\title{
FAULT ISOLATION IN NONLINEAR ANALOG CIRCUITS WITH TOLERANCE USING THE NEURAL NETWORK-BASED $L_{1}$-NORM
}

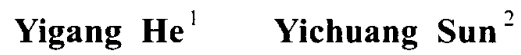 \\ 1. School of Electrical and Information Engineering, Hunan University, Changsha, 410082, P.R. China \\ E-mail address: vgherimail hunu.edu.cn \\ 2.Department of Electronic, Communication and Electrical Engineering, Faculty of Engineering and \\ Information Sciences, University of Hertfordshire, Hatfield AL10 9AB, U.K. \\ E-mail address: visunimerts ac uk
}

\begin{abstract}
This paper deals with fault isolation in nonlinear analog circuits with tolerance under an insufficient number of independent voltage measurements. A neural network-based $\mathrm{L}_{1}$-norm optimization approach is proposed and utilized in locating the most likely faulty elements in nonlinear circuits. The validity of the proposed method is verified by both extensive computer simulations and practical examples. One simulation example is presented in the paper.
\end{abstract}

\section{INTRODUCTION}

For nearly four decades, the subject of fault location in analog circuits has been of interest to researchers in circuits and systems. In recent years research of analog fault diagnosis has been further motivated by rapid development in analog VLSI signal processing. RF integrated circuits, mixed-signal testing and system on chip implementation. A number of promising techniques have emerged [1 5]. However, analog circuit fault location has proved to be an extremely difficult problem. This is mainly because of component tolerance and the nonlinear nature of the problem.

Among many fault diagnosis methods, the $\mathrm{L}_{1}$ optimization technique is a very important parameter identification approach [2-5], which is immune to tolerance. This method has been successfully used to isolate most likely faulty elements in linear analog circuits and combined with neural networks [6], real-time testing can be possible for linear circuits with tolerance [6].

Some fault verification methods have been proposed for nonlinear circuit fault diagnosis [2]. Based on these linearization principles, parameter identification methods can be developed for nonlinear circuits. In particular, the $\mathrm{L}_{\text {! }}$ optimization method can be extended and modified for fault diagnosis of nonlinear circuits with tolerance. Neural networks can also be used to facilitate the method to be more effective and faster for nonlinear circuit fault location.

This paper is to develop a neural network-based $L_{1}$-norm optimization technique for fault diagnosis of nonlinear analog circuits with tolerance.

\section{2. $L_{1}$-NORM OPTIMIZATION APPROACH FOR FAULT LOCATION OF NONLINEAR CIRCUITS}

Assume that a nonlinear resistive circuit has $\mathbf{n}$ nodes (excluding the reference node), $m$ of which are accessible. There are $b$ branches, of which $p$ elements are linear and $q$ nonlinear, $b=p+q$. The components are numbered in the order of linear to nonlinear elements. For simplicity, we assume that all nonlinear elements are voltage controlled, with characteristics being denoted as $i_{p+1}=f_{p+1}\left(v_{p+1}\right), \ldots, \quad i_{p+q}=f_{p+q}\left(v_{p+q}\right)$

When the nonlinear circuit is fault-free, the nonlinear component will work at its static point $Q_{0}$ and its voltage-current relation can be described as $i_{Q_{0}}=y_{0} v_{Q 0}$ where $y_{0}$ is the value of static conductance at working point $Q_{0}, i_{Q 0}$ and $v_{Q 0}$ are the current and voltage at point $Q_{0}$, respectively. When the circuit is faulty, no matter whether the nonlinear element is faulty or not, the static parameter will change from $y_{0}$ to $y_{0}+\Delta y$, where $\Delta y$ represents the increment from $y_{0}$. The change $\Delta y$ can be equivalently described by a parallel current source $v \Delta y$ where $v$ is the actual voltage $[2,3]$. For the linear elements, as is well-known,

0-7803-6685-9/01/\$10.00@2001 IEEE 
the change in a component value from its nominal can be represented by a current source. For a small signal excitation which lies in the neighborhood of its working point $Q_{0}$, the nonlinear resistive element can be replaced by a linear resistor. According to the superposition theorem, we can derive [2-5]

$$
\begin{aligned}
& \Delta V_{m}=H_{m b} E_{b} \\
& E_{b}=\left[e_{1}, e_{2}, \ldots, e_{h}\right]^{T} \\
& e_{i}=v_{i} \Delta y_{i}
\end{aligned}
$$

where $\Delta V_{m}$ is the increment vector of the voltages of accessible nodes due to faults, $H_{m b}$ is the coefficient matrix that relates the accessible nodal voltages to the equivalent current source vector $E_{k}$, which can be calculated from the nominal linear conductances and working point conductances of nonlinear components, $v_{i}$ is the actual branch voltage for the component $i, \Delta y_{i}(i=1,2, \ldots, p)$ is the change in the conductance of the linear component, and $\Delta y_{i}(i=p+1, \ldots, p+q)$ is the deviation from the static conductance of the nonlinear element.

Eq.(1) is an underdetermined system of linear equations for parameters $E_{h}$. Therefore the $\mathrm{L}_{1}$-norm optimization problem may be stated as:

$$
\operatorname{Minimize} \sum_{i=1}^{b}\left|e_{i}\right|
$$

Subject to $\Delta V_{m}=H_{m h} E_{b}$

The result of the optimization problem in Eq.(3) provides us with $E_{b}$, Then the network can be simulated using the external excitation source and obtained equivalent current sources $E_{h}$ to find $v_{i}, i=I, \ldots$, $b$ and $i_{i}$ for nonlinear components, $i=p+l, \ldots b$. The conductance change in every network component can be easily computed using Eq.(2b). Comparing the change in every linear component with its allowed tolerance, the faulty linear components can be readily located. For a nonlinear resistive element we need to further check the relation

$$
i_{Q 0}+\Delta i=\left(y_{0}+\Delta y\right)\left(v_{Q 0}+\Delta v\right)
$$

to determine whether the element is faulty or not, or in other words, whether or not the actual working point remains on the normal characteristic curve within tolerance [2-5]. If Eq.(4) holds within its tolerance, the nonlinear element is fault-free and the $\Delta y$, the result of working point $Q_{0}$ moving along its characteristic function curve are caused by other faulty elements. If Eq.(4) does not hold, the nonlinear element is faulty.

Eq.(3) is restricted to a single excitation. In fact, multiple excitations can be used to enhance diagnosability and provide better results. For $k$ excitations applied to the faulty network, the $\mathrm{L}_{1}$-norm problem is formulated as:

$\operatorname{Minimize} \sum_{i=1}^{b}\left|\Delta y_{i} / y_{i 0}\right|$

Subject to $\left[\begin{array}{c}\Delta V_{m}^{1} \\ \Delta V_{m}^{2} \\ \cdots \\ \Delta V_{m}^{k}\end{array}\right]=\left[\begin{array}{c}H_{m b}^{1} V_{h}^{1} \\ H_{m b}^{2} V_{h}^{2} \\ \cdots \\ H_{m h}^{k} V_{h}^{k}\end{array}\right] \Delta Y$

where

$$
V_{h}=\operatorname{diag}\left(v_{1}, v_{2}, \ldots, v_{b}\right) \text {. }
$$

$\Delta Y=\left[\Delta y_{1}, \Delta y_{2}, \ldots, \Delta y_{h}\right]^{\gamma}, \quad y_{i 0}(i=1, \ldots, p)$ represent nominal values of linear elements, and $y_{i 0}(i=p+1, \ldots, p+q)$ represent static conductances of nonlinear elements at working point $Q_{0}$. The superscripts $l$ to $k$ refer to different excitations. 
Traditionally, a linear programming algorithm is applied to solve the problem in Eq. (5). To preserve the linear relationship between $\Delta V_{m}$ and $\Delta Y$, the actual branch voltages $v_{i}, i=1, \ldots, b$ have to be assumed as known values. Therefore a repeated iterative procedure is needed, and its on-line computation is high. If the actual voltages $v_{i}$ are also regarded as the variables to be optimized, the values of $\Delta Y$ can be obtained after only one optimization process. In this case the $L_{1}$-norm problem can be stated as

Minimize $\sum_{i=1}^{b}\left(\left|\Delta y_{i} / y_{i 0}\right|+\left|\Delta v_{i} / v_{i 0}\right|\right)$

Subject to $\left[\begin{array}{c}\Delta V_{m}^{1} \\ \Delta V_{m}^{2} \\ \cdots \\ \Delta V_{m}^{k}\end{array}\right]=\left[\begin{array}{c}H_{n b}^{1} V_{b}^{1} \\ H_{m b}^{2} V_{b}^{2} \\ \cdots \\ H_{m b}^{k} V_{b}^{k}\end{array}\right] \Delta Y$

where $v_{i 0}$ represents the nominal branch voltage, $\Delta v_{i}$ is the change

in the voltage due to faults and tolerance, and $\Delta v_{i}+v_{i 0}$ the actual voltage $v_{i}$.

From Eq.(6) we can obtain $\Delta Y$. For a linear element if $\Delta y / y_{0}$ exceeds its allowed tolerance significantly, we can consider it to be faulty. But for a nonlinear resistive element, we cannot simply draw a conclusion. For analog circuits with tolerance, the relation of the voltage and current of a nonlinear resistive element can be represented by a set of curves instead of a single one due to tolerance, the nominal voltage to current characteristic of the nonlinear element being in the center of the zone. Therefore for a nonlinear component, after determining $\Delta y / y_{0}$ we need to simulate the nonlinear circuit again to judge whether the component is faulty or not. If the actual VI curve of the nonlinear element significantly deviates from the tolerance zone of curves, the nonlinear element can be considered as a faulty one. Otherwise, if the actual curve falls within the zone, the nonlinear element is faultfree.

\section{NEURAL NETWORKS APPLIED TO $L_{1}$-NORM FAULT DIAGNOSIS OF NONLINEAR CIRCUITIS}

According to the above analyses, nonlinear circuit diagnosis $\mathrm{L}_{1}$ norm problem has three representations corresponding to Eq. (3), (5) and (6), respectively. $\mathrm{L}_{1}$-norm problem Eq.(3) belongs to underdetermined linear equation parameter estimation problem, $\mathrm{L}_{1}$ norm problem Eq.(6) is the problem of nonlinear pararneter estimation. $\mathrm{L}_{1}$-norm problem Eq.(5).is solved traditionally by using linear programming algorithm, hence, $\mathrm{L}_{1}$-norm problem Eq.(5) can be considered as the problem of linear parameter estimation.

The $\mathrm{L}_{1}$-norm problem above can be transformed to unconstrained optimization problem using nondifferentiable exact penalty approach.. When the conditions for optimality of the nonlinear $\mathbf{L}_{1}$ norm problem are satisfied, the solution of the unconstrained problem will be that one the corresponding constrained $\mathrm{L}_{1}$-norm problem.

There exist many very efficient numerical algorithms to solving the least absolute value optimization problem[3].But one possible and very promising approach to solving such optimization problem in real time is to apply artificial neural networks[6].For solving the $\mathrm{L}_{1}$-norm problem above by using neural networks, the key step is to derive a computational energy function (Lyapunov function) $E(x)$ so that the lowest energy state will correspond to the desired solution. According to optimization theory, the objective function of the unconstrained optimization problem can be taken for computational energy function of neural network. Using a general gradient approach for minimization of $\mathrm{E}(\mathrm{x})$, the unconstrained optimization problem can be mapped to a system of differential equations. The system can be implemented by analog neuron-like networks which can find solutions in almost real-time. In order to limit space of the paper, the neural networks for solving the analog diagnosis $\mathrm{L}_{1}$-norm problem aren't included Actually, the algorithm and/or architecture of neural networks for solving the analog diagnosis $\mathrm{L}_{1}$-norm problem can be deduced from reference [4] and [6].

\section{EXAMPLE OF THE NEW METHOD FOR FAULT LOCATION OF NONLINEAR CIRCUITS}

Several examples have been simulated and a good agreement with theoretical considerations have been obtained for all of them. We are now presenting one example to see how the proposed method works with nonlinear circuits with tolerance.

Consider the nonlinear resistive network shown in Fig.1, with the nominal values of linear elements 1 to 6 being 


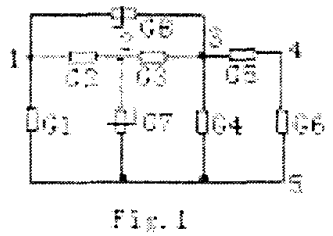

$y_{i t}=I(1=1, \ldots, 6)$ and the characteristics of nonlinear resistive elements 7 and 8 being $i_{7}=10 v_{7}^{3}$ and $i_{8}=5 V_{8}^{2}$, respectively. Both linear element parameters and static conductances $\left(y_{-0,}\right.$ and $\left.y_{* w}\right)$ of nonlinear elements are with tolerance of \pm 0.05 . Nodes $1,3,4$, 5 are assumed to be accessible with node 5 being taken as the ground. Node 2 is assumed to be internal where no measurement can be performed.

For a single small signal current excitation with $10 \mathrm{~mA}$ at node 1 , the changes in the accessible nodal voltages due to faults can be obtained as $\quad \Delta V_{m}=[0.0044,0.001,0.0004598]^{T}$. Construct the matrices required by Eq.(6) using the nominal/static component values and solve the $L_{1}$ problem in Eq.(6) using the neural network in [4] The neural network with $r_{i}^{*}=10$ and zero initial state has been simulated using the fourth-order Runge-Kutta method. The equilibrium point of the neural network is the solution of the $L_{1}$ problem, given by $\Delta y_{1} / y_{10}=-0.5071$. $\Delta y_{3} / y_{30}=-0.037 . \quad \Delta y_{7} / y_{70}=-0.43065$, $\Delta y_{8} / y_{80}=-0.4802, \Delta y_{i} / y_{i 0}=0, i=2.4 .5 .6$. Linear elements 2, 4, 5, 6 are normal as the conductance change is zero. The conductance change in linear element I significantly exceeds its allowed tolerance, therefore we can judge it is faulty. The change in linear elcment 3 slightly exceeds its allowed tolerance, but we can still consider it to be non-faulty. The changes in nonlinear element static conductances significantly exceed their allowed tolerances. We simulate the faulty nonlinear circuit again and find that only the VI curve of nonlinear element 7 significantly deviates from its tolerance characteristic zone, hence element 7 is faulty and element 8 is fault-free. In fact, in our original setting up, linear element 1 and nonlinear element 7 are assumed faulty. It can thus be seen that the proposed method can correctly locate the faults.

\section{CONCLUSIONS}

A neural network-based $\mathrm{L}_{1}$-norm optimization approach has been proposed for fault diagnosis of nonlinear resistive circuits with tolerance. An example has been presented, which shows that the proposed method can effectively locate faults in nonlinear circuits. The new method is suitable for on-line fault diagnosis of nonlinear circuits as it requires fewer steps in the $\mathrm{L}_{1}$ optimization and the use of neural networks further speed up the diagnosis process.

\section{ACKNOWLEDGEMENT}

This work is supported by the Natural Science Youth Funding Council of China under Grant No.59707002, Wang Kuancheng Education Funding Council, and Plan Aided Financially for Major Teachers of Colleges and Universities of Ministry of Education of China..

\section{REFERENCES}

[1] R.W. Liu, ed., Testing and diagnosis of analog circuits and systems, Van Nostrand and Reinhold, 1991.

[2] J. W.Bandler, R.M.Biernacki, A.E.Salama, and J. A. Starzyk, "Fault isolation in linear analog circuits using the L1 norm," Proc. IEEE Int. Symp. CAS, pp.1140-1143, 1982.

[3] J. W. Bandler, W. Kellermann and K. Madsen, "Nonlinear L] optimisation algorithm for design, modeling and diagnosis of networks," IEEE Trans. Circuits and Syst., vol. 34, no.2, pp 174-181, 1987.

[4] Y. G. He, X. J. Luo, and G. Y. Qiu, "A neural-based nonlinear $l$, norm optimization algorithm for diagnosis of networks," J. of Electronics, vol.15, No. 4, pp.365-371, 1998.

[5] Y. Sun and Z. X. Lin. "Fault diagnosis of nonlinear circuits," Journal of Dalian Maritime University, vol.12, No.1, pp.73.83, 1986.

[6] A. Cichocki and R. Unbchauen, Neural networks for optimization and signal processing, John Wiley, 1993, chapter 5-7. 